\title{
Surgery needs better support from EU
}

\author{
Nicola Di Lorenzo • Valeria Tognoni • \\ Luca Iezzi · Achille Lucio Gaspari
}

Published online: 14 January 2011

(C) Springer Science+Business Media, LLC 2011

According to the World Health Organization (WHO) [1], approximately 234 million major surgical operations are conducted each year. This includes more than 63 million people who undergo surgery to treat traumatic injuries, 31 million for treating cancers, and another 10 million for pregnancy-related complications. Sooner or later, most of world's population will have to go under the knife, expecting to be treated with the latest technology and the most effective procedures.

Studies conducted by the WHO [1] conclude that complications after surgery result in disability or prolonged stay in 3-25\% of hospitalized patients. Moreover, iatrogenic lesions represent the seventh cause of death in western countries (approximately 100 patients/day in the United States), with surgeries "contribution" of 50\%. This means that at least 7 million patients annually have postoperative complications and indicates the need to investigate and develop better surgical procedures, instruments, and training programs to offer a greater potential to solve many healthcare problems and complications.

Since the advent of the European Union (EU), research in the medical field has a relevant place in the Union's activities. Every year the EU devotes relevant funds to healthcare research by means of Framework Programs (FP) and other actions. FP7, acting 2007-2013, is distributing grants for 6.1 billion euro-approximately $700 \mathrm{M}$ in 2011 .

The main efforts are devoted to genomics, immunotherapy and vaccines, stem cells, gene therapy, prosthesis, tools for modern biology, imaging, detection, and analytical tools; moreover, translational research in major infectious

N. Di Lorenzo $(\bowtie) \cdot$ V. Tognoni · L. Iezzi · A. L. Gaspari Dipartimento di Scienze Chirurgiche, Università degli studi di Roma "Tor Vergata", Rome, Italy

e-mail: nicola.di.lorenzo@uniroma2.it diseases and other diseases, such as cardiovascular ones, cancer, diabetes, and obesity. Within this scenery, surgery is participating with a small presence, although it is now considered the "gold standard" treatment for many of the above-mentioned pathologies (cancer, metabolic syndrome, cardiovascular diseases), with significant results reported for short- and long-term outcomes.

Although the cornerstones of medical research and future perspectives, we cannot deny that drug pipelines are drying up and gene therapy has yet to deliver the anticipated breakthroughs. On the other hand, surgical procedures and innovative medical technology can offer a greater potential to solve many healthcare issues. They also can provide, in many cases, a viable alternative to lengthy drug-based treatments and costly mobility aid systems, which can easily last a patient's lifetime, for example, gastroesophageal reflux disease and morbid obesity.

Even if European citizens are daily dealing with surgical experience, there is one aspect of healthcare in which surgeons lag behind other healthcare professionals: financial support for research in basic and clinical innovation and advancements.

For example, only $1.3 \%$ of U.K. government funding for medical research is spent on surgery research [2].

Clearly, there is a low level of awareness of surgery amongst policy and decision-makers, which needs to be addressed; consequently, interest in supporting surgery in EU programs and policies has to increase.

Surgery must be included as a key dimension to improving the quality of life of European citizens and contribute to ameliorate the service that health systems provide in terms of costs, efficacy, and efficiency. More specifically, surgery needs to be included as a priority in the final part of FP7 and in the Health priorities of the Eight Research Framework Program (FP8). 
Since last year, the principal author has started activities (including a seminar held at the EU parliament) and established several contacts with representative of the EU commission to address the importance of funding for surgery and to claim for a better attention to surgical profession, with a greater contribution for research in surgery. The surprising answer to these actions, from the EC officers, was that it was the first time that this problem has been brought to their attention!

This makes it clear that a certain arrogance, which characterizes somehow our professional figures, has the worst effect in a world ruled by imaging and marketing. On the other hand, a busy practice discourages surgeons to spend their time lobbying for their own fundraising. A third reason could be identified in the fragmentation of the European surgical community: some international societies represent single specialties, but there is not an institution comparable to the American College of Surgeons (ACS) in terms of representativeness and impact on relationship with political and regulatory institutions.

\section{Surgery's relevance for EU health systems}

A breakthrough event hit healthcare systems in the early $1990 \mathrm{~s}$; minimally invasive surgery changed dramatically the pain and outcome of many surgical treatments and brought many other advancements on the pathway of innovative therapies. Europeans led this revolution, being the first to perform most interventions, to introduce remote surgery, and to develop new access to the abdominal cavity [3, 4].

At the same time, the work of surgeons has supported progress in other important medical areas. This has probably been facilitated by the pertinence of surgery to an interdisciplinary area of research constantly drawing on technologies, tools, and materials, although in many cases they may have not been initially intended to be used in surgical procedures. The crossover with other disciplines within and outside the life sciences continues to be considerable and will become even more important in the future. Here are some examples: (1) surgical resection of gastrointestinal segments has led to the discovery and understanding of enterohormonal patterns that determine patients' recuperation rates after, for example, obesity, diabetes, or pancreatic interventions; (2) the development of organ transplantation has brought better understanding about how the human immune system reacts in terms of rejection or acceptance; and (3) biocompatibility of a great number of prosthetic materials has been definitively assessed by clinical use in cardiovascular, orthopedic, otologic, and general surgery. These are only few examples of a large number of research applications of surgery during the past years.
An examination of the projects supported under the seventh framework program (FP7) shows that there is a long way to go to place surgery at the forefront. Currently, there are only 33 funded projects that are related or have something to do with surgery. However, only six of these projects are within the work program for FP7 Cooperation Theme Health. The majority of investigations are currently being conducted within the ICT Theme, and most of them are exclusively focused on new imaging systems and virtual simulation. Surgery-led research seems to have lost its role as the driving force behind those breakthrough applications [5]. On the other hand, the same FP7 Cooperation Theme Health includes more than 30 projects related to research in genomics or genetics, and more than 100 regarding molecular studies and drugs.

In currently funded projects, surgery has become the end rather than the point of departure. It is therefore necessary to stimulate research and development (R\&D) that is led by surgery from the beginning. Interdisciplinarity can be highly beneficial, especially in such a wide field, but policy makers and research managers must understand the requirements of surgeons and patients, who, at the end of the day, are the ones entering the operating room. The risk of not doing so raises the perspective of developing applications that the professional community may not embrace.

\section{Proposals}

In 2009, the EU commission notified that research would be funded by focusing the choice on three main pillars: "Biotechnologies, generic tools and medical technologies for human health"; "Translating research for human health"; and "Optimizing the delivery of health care to European citizens."

Each of them contains topics that are strictly connected to surgery as "Innovative therapeutic approaches and interventions" or "Research on major diseases" and again "Translation of clinical outcome into clinical practice." We would have expected to find, in the Health Research Work Program for 2010, a larger space for research in surgery, whereas it is cited only twice in a 49-page document. Rumors concerning the 2011 draft say that it is more liberal regarding the investigator-driven proposal, but until today there seems to be no specific measure devoted to surgeons' leadership.

In contrast to this scenery, which shows a very low amount of resources in the matter of funds assigned to surgery by EU, there are many innovative fields of research that deserve substantial grants. Regarding biotechnologies, there are innovative therapeutic approaches and interventions that must be developed; first of all, natural orifice transluminal surgery (NOTES) is one of the most promising 
areas of research within the thematic priority of noninvasive or minimally invasive approaches. This revolutionary technique, when applied on a broad base, could offer many advantages: lower anesthesia dosage; faster recovery time; shorter hospital stay and therefore lower cost; less pain for the patient; and better aesthetic results due to the lack of visible scars. Applications could include battlefield surgery to wounded soldiers and operations in areas where establishing a sterilized setting would be difficult.

Public institutions, such as university and research centers, and private companies usually combine the resources in matters of money and human assets to generate consortiums, which guarantee a number of very qualified experts focused on the developing of innovative projects.

The EURONOTES Foundation was established by the two major European societies active in the field: European Association for Surgical Endoscopy (EAES) and European Society of Gastrointestinal Endoscopy (ESGE). It is coordinating the most research about NOTES activities. It received small grants, for a total amount of 500.000 Euros, thanks to private companies support. The corresponding entity of EURONOTES, named NOSCAR Consortium, received in $2007 \$ 2$ million in grants from medical device manufactures to conduct research, and funds are almost doubled in 2009, which does not include funding from $\mathrm{NIH}$ [6].

It is clear that Europe will not be able to be competitive with this lack of resources, even if we need to remember that the first NOTES procedure on humans was conducted in Europe within the ANUBIS operation in April 2007.

Even though there have been innovations in other forms of therapy, surgery remains an indispensable remedy for most nontransmittable diseases, such as cancer and cardiovascular diseases. Recent research also has shown that surgery might be an effective cure against metabolic syndrome [7] and diabetes [8, 9], by decreasing long-term mortality and morbidity in obese patients. Christou et al. [10] showed a reduction in the relative risk of death by $89 \%$ for those patients who underwent surgery compared with control subjects who received medical treatment only as diet, physical activity, and medications.

Costs related to the single disease also must be considered. Morbid obesity costs the Italian institutions approximately 8.1 billion Euros per year, approximately $1700 €$ for a single obese subject; during his entire life he would cost the institutions $100.000 €$ more than a normal subject [11].

Innovative therapies for diabetes have even been addressed as a possible cure for Alzheimer's disease [12]; this would definitively be a field of great interest considering the aging European population.

In the field of optimizing the delivery of healthcare to European citizens, the third pillar mentioned by the EU is crucial: the translation of results of clinical research outcome into clinical practice. Research about the necessary capacities to adopt innovative alternatives along with the implementation of surgical audit programs would be highly desirable. Comparisons of surgical outcomes among different countries are difficult not just because of the data, but also because of the introduction of new surgical methods, which are not always performed according to standardized protocols. Furthermore, some operative methodologies are based on routines that have never been subject to evidence-based studies.

For that reason, it is crucial to introduce and register surgical interventions in a systematic and standardized way. Technology can play a key role in recording the nature of operations to enable practitioners all around the world to learn, correct, and improve their results. Collecting and measuring this type of information to develop procedural standards will help to reduce errors, design better curricula and portfolios for surgeons in training, and reduce the learning time.

The same principle should be applied to clinical trials. They play a very important role, ensuring that the new surgical procedure and tools can be safely used on humans. Currently, the investments in research activities represent approximately $1.8 \%$ of the European Gross Domestic Product compared with 2.8 and $2.9 \%$ of the United States and Japan, respectively [13].

Currently in Europe, most clinical trials are financed by private companies that are interested in supporting the introduction of novel products or techniques. In United States, the National Institutes of Health (NIH) in 2009 received $\$ 10.4$ billion provided by ARRA and funded $\$ 1.3$ billion for National Centers for Research Resources. Within the NIH, the Clinical Trial Planning Grant Program (R34) supports the development of Phase III clinical trials [14].

It must be stressed that a sponsored trial has a threefold more frequently favorable outcome compared with independent trials. Correcting these data according to Gilbert's criteria, sponsored trials report $74 \%$ favorable results for new devices and techniques, compared with $53 \%$ in trials sponsored by nonprofit organizations.

This results into a lack of credibility for European clinical trials. If the EU wants to remain competitive in the field of surgical innovations, new mechanisms to conduct surgical clinical trials must be put into place.

\section{Economics}

Surgery is one of the most important applications for medical technology and therefore a key element of competitiveness for the European economy. The European medical technology industry already invests $€ 5.8$ billion in R\&D and employs nearly 529,000 highly skilled workers. 
There were approximately 11,000 medical technology companies in Europe in 2005. More than $80 \%$ of them are small and medium enterprises (SMEs). FP7 already recognizes their importance and actively seeks to engage them in project participation. SMES working in the field of surgical applications and devices could benefit enormously from those changes if surgery is given the appropriate recognition in future research programs.

A market-based approach should always be balanced with the public financial support for pure surgical R\&D. Manufactures and health-related private companies only perform specific research programs to develop with new techniques and technologies that can be profitable. However, that does not guarantee the development of the necessary basic research to advance in fields that do not have such a high sales potential. This is precisely one more reason why there is an urgent need to incorporate surgical R\&D into the EU's health policy agenda and related research programs.

\section{Conclusions}

Surgery is a very fragmented field, divided into many disciplines, and it is getting even more dismembered because technological advances that make certain interventions easier have opened the doors to other medical practitioners and technicians who, in the past, were not able or certified to perform surgery. There is an urgent need for surgery to act with just one voice so that research needs can be clearly defined and EU global support mechanisms for surgery can be implemented. It is very important to establish connections among all those professionals who work in the operating room, especially among those institutions that have achieved a high level of professionalism and performance.

EAES can play a determinant role in this effort, having definitively conquered a predominant position in the European landscape of surgical societies and a leading role both in exploitation of surgical innovations and in educational activities. Recently, the association's leadership has met the EC at directories' level and further steps have been planned. Let's work together!

\section{References}

1. Ten facts on safe surgery (2008) WHO. Available at: http://www.who.int/features/factfiles/safe_surgery/en/. Accessed January 2008

2. Financial Times, 28 May 2009, p. 11

3. Marescaux J, Leroy J, Gagner M, Rubino F, Mutter D, Vix M, Butner SE, Smith MK (2001) Transatlantic robot-assisted telesurgery. Nature 413(6854):379-380

4. Marescaux J, Dallemagne B, Perretta S, Wattiez A, Mutter D, Coumaros D (2007) Surgery without scars: report of transluminal cholecystectomy in a human being. Arch Surg 142(9):823-826

5. Available at: http://cordis.europa.eu/fetch?CALLER=FP7_PROJ_ EN\&QZ_WEBSRCH=surgery\&QM_EP_PGA_A=FP7-HEALTH $\&$ USR_SORT $=E N \_Q V D+C H A R+D E S C$. Accessed January 2009

6. Tina Peng. Open Wide. No, Wider (2008) Newsweek, Available at: http://www.newsweek.com/id/130600. Accessed August 2008

7. Scopinaro $\mathrm{N}$ et al (2005) Specific effects of biliopancreatic diversion on the major components of metabolic syndrome: a long-term follow-up study. Diabetes Care 28:2406-2411

8. Scopinaro $\mathrm{N}$ et al (2008) A comparison of a personal series of biliopancreatic diversion and literature data on gastric bypass help to explain the mechanisms of resolution of type 2 diabetes by the two operations. Obes Surg 18(8):1035-1038

9. Rubino F, Gagner M (2002) Potential of surgery for curing type 2 diabetes mellitus. Ann Surg 236(5):554-559

10. Christou NV, Sampalis JS, Liberman M, Look D, Auger S, McLean AP, McLean LD (2004) Surgery decreases long-term mortality, morbidity, and health care use in morbidly obese patients. Ann Surg 240(3):416-423

11. Buchwald H, Estok R, Fahrbach K, Bauel D, Jensen MD, Paries WJ, Bantle JP, Sledge I (2009) Weight and type 2 diabetes after bariatric surgery: systematic review and meta-analysis. Am J Med 122(3):248-256.e5

12. Brain surgery treats Alzheimer's (2004) BBC News. Available at: http://news.bbc.co.uk/2/hi/health/3662231.stm. Accessed January 2008

13. Eurostat 2008, p. 195

14. Diabetes in Europe (2004) Towards a European framework for diabetes prevention and care, Workshop Proceedings, Dublin, 2004, p 7 